Ann. Biol. anim. Bioch. Biophys., I973, 13 (I), I7-24.

\title{
INFLUENCE DE LA TAILLE DES BLASTOCYSTES \\ ET DE LEUR CONSOMMATION D'OXYGENE SUR LEUR DÉVELOPPEMENT APRES TRANSPLANTATION CHEZ LA LAPINE
}

\author{
Suzanne WINTENBERGER-TORRÈS \\ avec la collaboration technique de Nicole VERMEIRE \\ Station centrale de Physiologie animale, \\ Centre national de Recherches zootechniques, I. N.R. A., \\ 78350 Jouy en Josas
}

\section{RÉSUMÉ}

La viabilité des blastocystes de lapine à cinq jours et demi post coitum a été évaluée d'après la taille atteinte à ce stade, la rapidité de croissance en culture in vitro, et la consommation d'oxygène.

La transplantation des blastocystes étudiés, dans des lapines receveuses gestantes de cinq jours et demi a donné seulement $26 \mathrm{p}$. Ioo de survie embryonnaire à 28 jours de gestation. La forte mortalité est due aux manipulations que l'on fait subir aux blastocystes transplantés et à l'ouverture de l'utérus.

La survie est significativement meilleure pour les blastocystes de grande taille et pour ceux qui ont la plus forte consommation d'oxygène. (La consommation d'oxygène étant exprimée par un coefficient : $\frac{\text { quantité d'O } \mathrm{O}_{2} \text { consommé }}{\text { taille }}$.

Chez la Lapine, des différences dans la vitesse de segmentation des œufs se manifestent dès le $2^{\mathrm{e}}$ jour. A cinq jours et demi post coitum, $20 \mathrm{p}$. Ioo des $\mathrm{I} 037$ blastocystes récupérés chez des lapines néo-zélandaises ont un diamètre $\leqslant$ à 1,75 $\mathrm{mm}$, tandis que la taille des autres peut atteindre $3,5 \mathrm{~mm}$.

De tels retards avant l'implantation peuvent expliquer une partie de la mortalité embryonnaire.

HAFEZ (I962) constate une différence significative des taux d'implantation avec des œufs de lapines transplantés au même âge, mais les uns étant à 7 blastomères et les autres à I5. La différence est en faveur des œufs à segmentation rapide.

Gates (I965) et Dickson (r966) ont constaté également chez la Souris des 
différences naturelles de vitesse de segmentation des œufs qui compromettent la survie embryonnaire.

Nous avions cherché à disposer de critères permettant d'évaluer le niveau métabolique des blastocytes :

- soit en mesurant la croissance en culture in vitro pendant 4 heures (WINTENBERGER-TORRÈS et DANEL, I97I),

- soit en enregistrant la consommation d'oxygène in vitro (WINTENBERGERTORRÈs, I970).

Dans le présent travail, nous avons appliqué ces deux techniques à des blastocystes de taille différente, avant de les transplanter dans des receveuses pour connaître leur viabilité.

\section{MATÉRIEL， ET MÉTHODES}

Les transplantations ont 'été effectuées chez des lapines receveuses anesthésiées. L'utérus est đégagé, on pratique une petite incision à environ $1,5 \mathrm{~cm}$ de la jonction utéro-tubaire ; le blastocyste est introduit à l'aide d'une pipette de verre et on referme l'ouverture par un point de suture.

Pour la collecte et le transfert des blastocystes, on a utilisé comme milieu, du I99 modifié (Milieu I) comprenant :

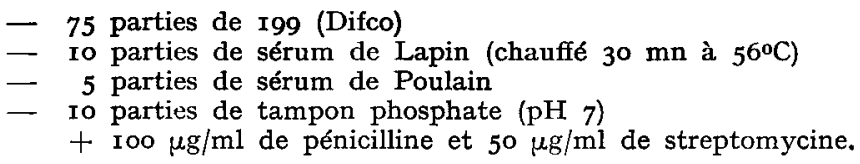

La durée entre l'abattage de la donneuse et l'introduction dans l'utérus de la reveceuse n'a pas excédé 30 minutes.

La mortalité embryonnaire après transfert des blastocystes normaux à cinq jours et demi est évaluée à 63 p. roo par STAPles (I970), ADAMs (I970) obtient, après transfert des blastocystes. provenant de morulas cultivées pendant 72 heures, $20,5 \mathrm{p}$. Ioo de jeunes.

Compte tenu de cette mortalité importante, nous avons voulu déterminer dans notre étude, la part d'échecs qui revient à l'intervention sur l'utérus et celle qui revient aux différentes manipulations auxquelles sont soumis les blastocystes, en réalisant trois séries d'expériences :

$$
\text { A. - Lot témoin } 1 \text { (fig. I) }
$$
fertile.

Quinze lapines Néo-Zélandaises sont opérées $\mathrm{r}_{42}$ heures après accouplement avec un mâle

On injecte dans l'utérus uniquement $0,5 \mathrm{mI}$ du milieu $\mathrm{I}$.

Les lapines sont sacrifiées les 28 ou $29^{\mathbf{e}}$ jour de la gestation. La survie embryonnaire est exprimée par le rapport entre le nombre de jeunes vivants et le nombre de corps jaunes.

La mortalité postérieure à l'implantation est exprimée par le rapport du nombre total de traces d'implantations dégénérées sur le nombre de corps jaunes.

\section{B. - Lot témoin 2}

Cinquante-huit blastocystes sont transférés dans 29 lapines receveuses déjà gravides. Les lapines receveuses Néo-Zélandaises ont été accouplées avec un mâle Fauve de Bourgogne domi-

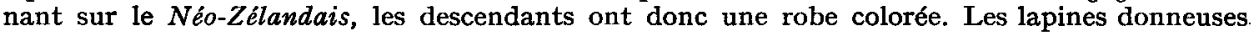
Néo-Zélandaises sont accouplées en même temps que les receveuses, avec un mâle $N e ́ o-Z e ́ l a n d a i s$ donnant par conséquent des jeunes blancs. 6 jours après, l'utérus des donneuses est perfusé dans. une étuve à $37^{\circ} \mathrm{C}$ avec le milieu I. Les blastocystes sont mesurés au micromètre oculaire (grossis - 
sement $\mathrm{I60}$ ). La taille est représentée par la moyenne de deux diamètres perpendiculaires. On choisit deux blastocystes de même taille ou de taille très voisine qui sont introduits dans l'u térus de la receveuse selon la même technique que dans le groupe $\mathbf{I}$.

Les lapines receveuses sont sacrifiées à 28-29 jours comme précédemment.

RECEVEUSE
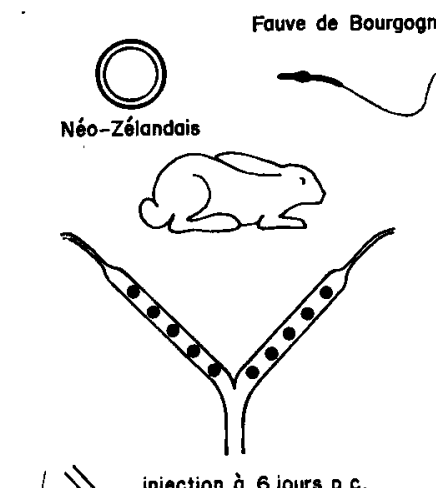

H

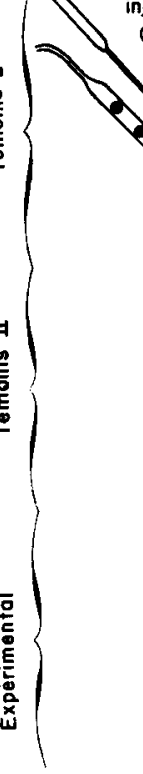
$0,5 \mathrm{ml}$ de 199

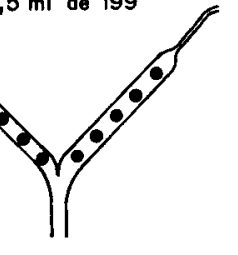

DONNEUSE<smiles>c1ccccc1</smiles><smiles>CCCCCC</smiles>

Néo - Zélandais

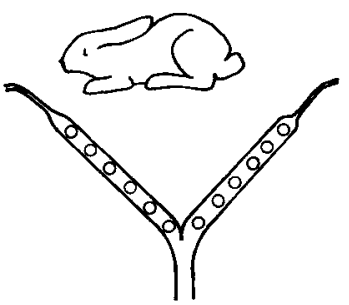

abattue
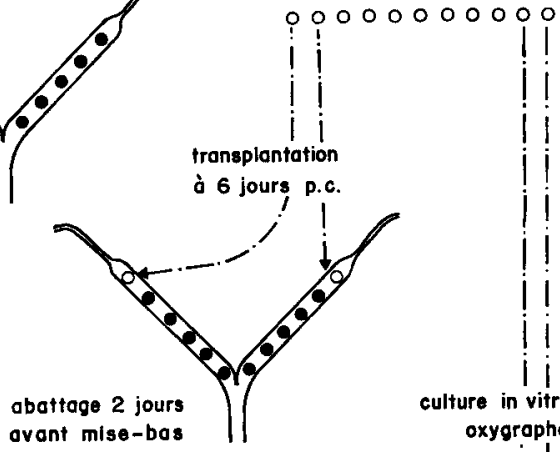

culture in vitro + oxygraphe

abottoge 2 jours ovant mise-bas

FIG. I. - Schéma du protocole expérimental

\section{C. - Lot expérimental 3}

Soixante et une lapines donneuses Néo-Zélandaises sont accouplées avec un mâle NéoZélandais et abattues cinq jours et demi ( 136 heures) après accouplement.

Les blastocystes sont mis en culture dans le milieu II composé de :

- 98 parties de sérum de Veau foetal frais ou lyophilisé ;

- 2 parties de tampon phosphate $(\mathrm{pH} 7)$ additionné de $100 \mu \mathrm{g}$ de pénicilline.

Après 4 heures de culture on choisit deux blastocystes de même taille dont on mesurera la consommation d'oxygène et qui seront transplantés dans des receveuses identiques à celles du 
lot témoin 2. Les cultures et mesures étant faites dans le sérum de Veau, nous avons jugé utile de laver les blastocystes dans le milieu I avant de les transplanter, afin d'éliminer le sérum de Veau, et les replacer ainsi dans les mêmes conditions que dans le lot précédent.

\section{RÉSULTATS}

\section{Témoin 1}

L'excision de l'utérus et l'injection d'une très faible quantité de milieu de culture perturbe de façon très appréciable la survie des embryons puisque $52,87 \mathrm{p}$. Ioo seulement arrivent à terme (tabl. I). La mortalité embryonnaire est nettement

TABLEAU I

Ripartition de la survie et de la mortalité embryonnaire dans le lot témoin 1

\begin{tabular}{|c|c|c|c|}
\hline \multicolumn{4}{|c|}{ Survie embryonnaire : Témoin 1} \\
\hline \multirow{2}{*}{$\begin{array}{l}\text { Nombre } \\
\text { de lapines }\end{array}$} & \multirow{2}{*}{$\begin{array}{c}\text { Nombre } \\
\text { de corps jaunes }\end{array}$} & \multicolumn{2}{|c|}{ Fortus vivants } \\
\hline & & Nombre & P. 100 \\
\hline 15 & 174 & 92 & 52,87 \\
\hline
\end{tabular}

Répartition de la mortalité : Témoin 1

\begin{tabular}{c|c|c|c}
$\begin{array}{c}\text { Mortalité totale } \\
(\%)\end{array}$ & \multicolumn{2}{|c|}{$\begin{array}{c}\text { Mortalité post-implantatoire } \\
\begin{array}{c}\text { Nbre de placentas } \\
+ \text { sites }\end{array}\end{array}$} & $\begin{array}{c}\text { Mortalité } \\
\text { préimplantatoire } \\
(\%)\end{array}$ \\
\hline 47,13 & 45 & 25,86 & 21,26 \\
\hline
\end{tabular}

plus élevée que le chiffre de $30 \mathrm{p}$. Ioo habituellement accusé chez la Lapine. Elle survient dans une proportion équivalente avant et après l'implantation et se trouve ainsi supérieure, avant l'implantation, à la proportion de 8,1 à 9,7 p. Ioo signalée pat Adams (1960).

\section{Témoin 2}

La survie des blastocystes transplantés, qui donnent des jeunes blancs, a été comparée dans les deux derniers lots (tabl. 2). La transplantation par elle-même, est suivie de la mort de 46,5 p. Ioo des blastocystes. La situation se trouve encore aggravée dans le lot expérimental, où les blastocystes ont été manipulés in vitro, et 
la mortalité atteint 74 p. Ioo. La différence entre les deux pourcentages est hautement significative. Ces manipulations compromettent donc fortement la survie après transplantation.

\section{TABLEAU 2}

Résultats des survies après transfert des blastocystes dans les lots témoin 2 et expérimental

Survie des blastocystes "blancs " transférés

\begin{tabular}{|c|c|c|c|c|c|}
\hline & \multirow{2}{*}{$\begin{array}{c}\text { Nombre total } \\
\text { de blastocystes } \\
\text { transférés }\end{array}$} & \multicolumn{2}{|c|}{ Fotus vivants } & \multicolumn{2}{|c|}{ Mortalité } \\
\hline & & Nombre & P. 100 & Nombre & P. 100 \\
\hline Témoin 2 & 58 & 31 & 53,5 & $\therefore 7$ & 46,5 \\
\hline Expérimental. & 123 & 32 & 26 & 91 & $71^{\prime}$ \\
\hline
\end{tabular}

Il est intéressant de mettre en parallèle la survie des embryons " noirs " dans les deux derniers lots. L'apport d'un blastocyste étranger n'a pas d'influence sur l'avenir des blastocystes qui sont déjà dans la corne utérine (tabl. 3, témoin 2); la mortalité est sensiblement la même que dans les témoins I $(47,6 \mathrm{p}$. Ioo contre $47, \mathrm{I} 3$ p. Ioo) et due, comme nous l'avons vu, à l'intervention sur l'utérus. Par contre, la mortalité élevée des blastocystes soumis aux tests de croissance et de respiration agit significativement sur la survie des blastocystes " noirs" in situ.

\section{TABLEAU 3}

Survie des blastocystes présents dans les cornes utérines des lapines receveuses dans les lots témoin 2 et expérimental

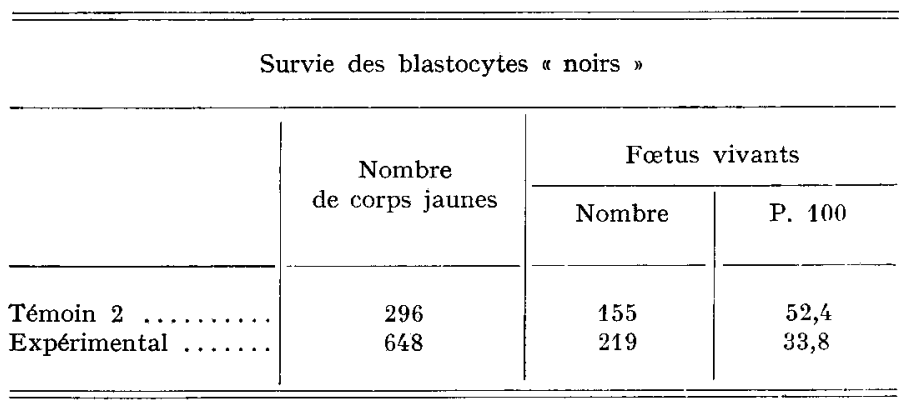

En groupant les résultats des lots 2 et 3 on peut comparer les pourcentages de survie et de mort des blastocystes transplantés (" blancs") en fonction de la survie des blastocytes " noirs " in situ.

La mortalité dans le lot témoin I atteint en majorité les embryons de la moitié supérieure de la corne utérine ce qui semble logique puisque l'incision de l'utérus 
se situe à environ I $\mathrm{cm}$ de la jonction utéro-tubulaire. L'augmentation de mortalité constatée dans les lots témoin 2 et expérimental se traduit par une extension des dégénérescences réparties sur toute la longueur de la corne, voire même à une dégénérescence totale de tous les embryons situés à l'intérieur d'une même corne.

\section{Survie des blastocystes en fonction des critères étudiés}

a) Relation taille/survie.

Dans le lot témoin 2 la moyenne de taille des blastocystes transplantés qui survivent (fig. 2, blanc vivant) est de $2,80 \mathrm{~mm}$, tandis que celle des blastocystes qui dégénèrent est de $2,62 \mathrm{~mm}$. La différence n'est pas significative, mais est en faveur des plus gros blastocystes.
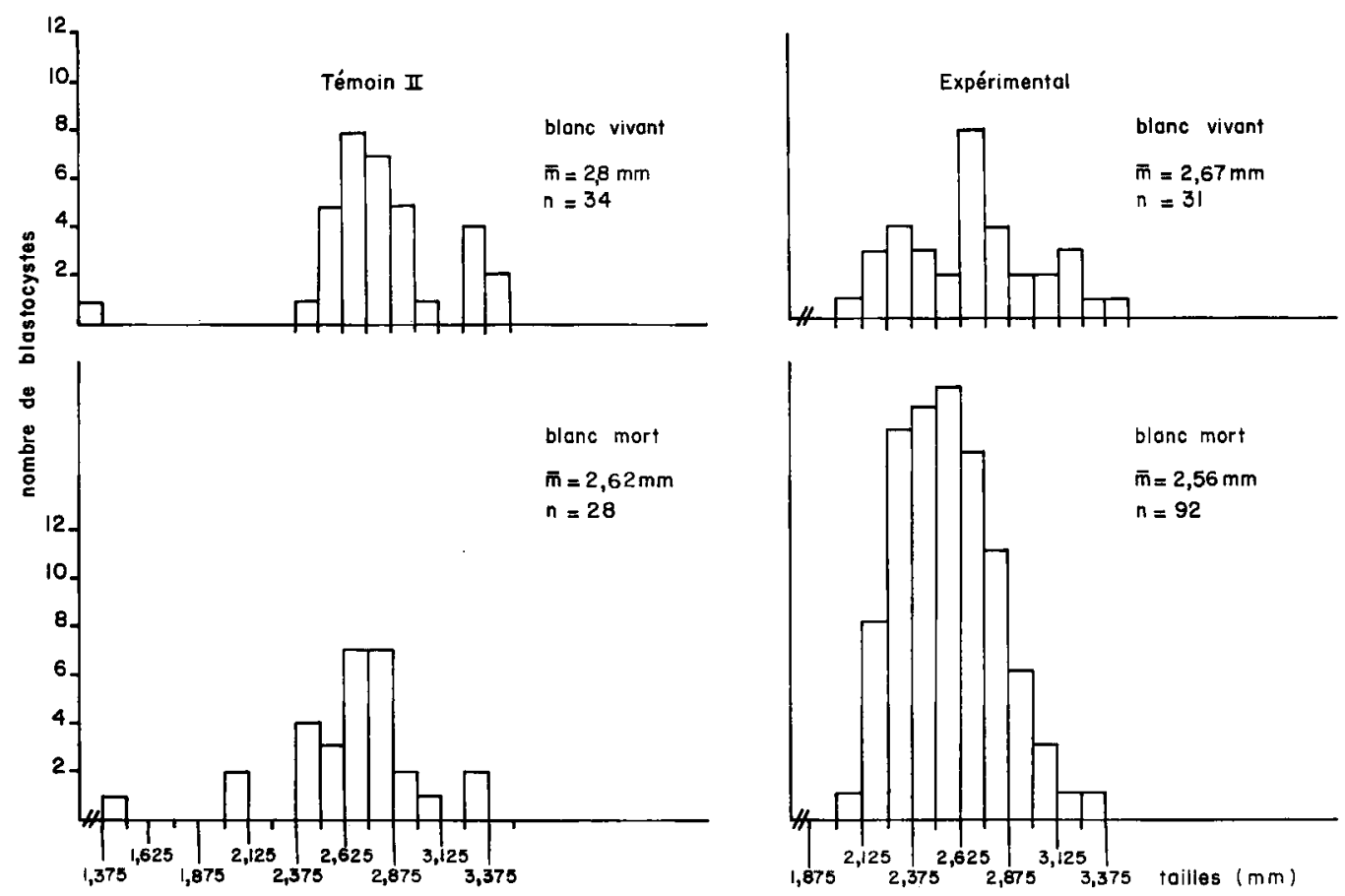

FIG. 2. - Repartition des tailles des blastocystes destinés à survivre ou d mourir dans les lots témoin 2 et expérimental

Dans le lot expérimental la moyenne de taille des blastocystes transplantés qui survivent est $2,67 \mathrm{~mm}$ et celle des blastocystes dégénérant est $2,56 \mathrm{~mm}$; la survie est significativement meilleure quand on transplante des blastocystes plus gros.

b) Relation croissance/survie.

La croissance in vitro est exprimée par le rapport :

$$
\frac{\mathrm{T}_{4}-\mathrm{T}_{0} \times \text { roo }}{\mathrm{T}_{0}}
$$


Ann. Biol. anim. Bioch. Biophys., I972, 12 (4).

\title{
ERRATUM
}

\section{INFLUENCE DE L'ACIDE CAPRYLIQUE SUR LE MÉTABOLISME DES AUTRES ACIDES GRAS CHEZ LE RAT EN CROISSANCE}

\author{
B. AUROUSSEAU et L. DE GROOT \\ Laboratoire d'Études des Métabolismes, \\ Centre de Recherches de Clermont Ferrand, I. N. R. A. \\ 63110 Clermont Ferrand
}

Tableau 6, page 625 .

En raison de la dilution des foies dans deux fois leur poids d'eau lors de leur homogénéisation avant extraction, il faut multiplier par 3 (facteur correctif connu à $\mathrm{O}, \mathrm{I}$ p. Ioo près) les chiffres relatifs à la teneur pondérale des foies en AG. 

$T_{0}$ étant la taille au moment de la mise en culture et $T_{4}$ la taille après 4 heures de culture.

Les résultats sont rapportés sur le graphique 3 . Il n'y a pas de différence significative entre la croissance moyenne des blastocystes destinés à survivre et celle des blastocystes dégénérant.

c) Relation consommation d'oxygène/survie.

Pour comparer les consommations d'oxygène entre blastocystes de taille différente, nous avons calculé le quotient : consommation d'oxygène/taille des blasto-

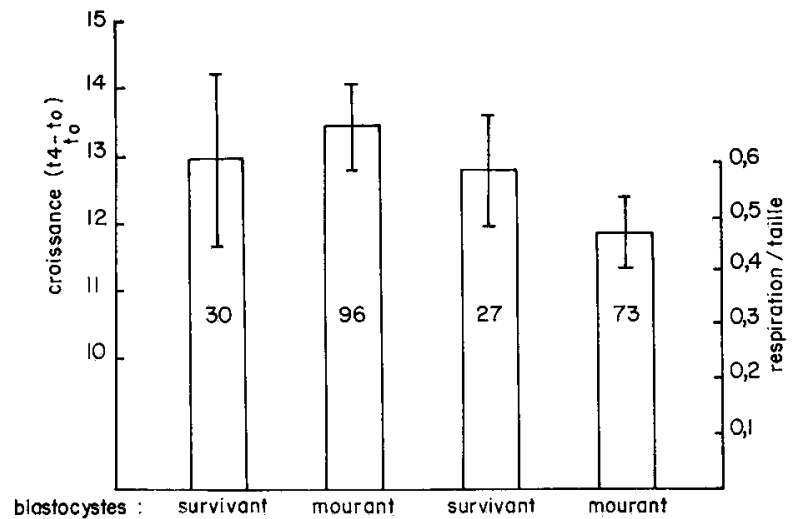

FIG. 3. - Histogramme établissant la moyenne de croissance et la moyenne du quotient:

$\frac{\text { Consommation } \mathrm{d}^{\prime} \mathrm{O}_{2}}{\text { taille }}$ des blastocystes destinés à survivre ou à dégénérer, dans le lot expérimental

cystes. Les moyennes de ces quotients établies pour les blastocystes qui se développent et ceux qui meurent montrent que les valeurs sont significativement supérieures pour les blastocystes qui survivent (fig. 3).

\section{DISCUSSION}

Les résultats de ces expériences ne permettent pas de déterminer une limite de taille des blastocystes en dessous de laquelle la dégérescence se produit automatiquement.

Cela s'explique en partie par le fait que, pour des raisons de sensibilité de l'oxygraphe, nous avons opéré un choix dans les blastocystes transplantés, ne prenant que ceux dont le diamètre moyen était égal ou supérieur à $\mathrm{I}, 30 \mathrm{~mm}$. On a donc éliminé les blastocystes ayant peut-être les plus grandes chances de dégénérer.

L'importance de la mortalité embryonnaire provoquée par l'incision de l'utérus a réduit considérablement les écarts entre les résultats. La perte subie pour des transplantations à ce stade de la gestation, est du même ordre que celle accusée par STAPLE (I97I). 
L'existence d'une suture au sommet des cornes utérines, posée au moment de la transplantation, pourrait être responsable des dégénérescences embryonnaires. Cependant, la taille de cette ligature la rend difficilement assimilable à un " IUD » et ADAMs ( 1965 ) ne trouve un effet inhibiteur des sutures que lorsqu'une extrémité du fil de soie est laissée libre à l'intérieur de la corne utérine. Nous n'avons cependant pas recherché la présence de leucocytes polymorphonucléaires dans l'utérus lorsqu'il y avait eu des dégénérescences.

D'autre part, le sommet des cornes, choisi comme lieu de transplantation, pour éviter l'expulsion des blastocystes de l'utérus vers le vagin, n'est pas particulièrement favorable à l'implantation chez la Lapine comme chez la Ratte.

Il faut retenir cependant, que les blastocystes à développement plus rapide in vivo, ont une probabilité de survie meilleure, et que, l'activité métabolique évaluée par la consommation d'oxygène, donne une indication valable du potentiel de développement embryonnaire.

Reçu pour publication en jillet 1972.

\title{
SUMMARY
}

\author{
EFFECT OF BLASTOCYST SIZE AND OXYGEN CONSUMPTION \\ ON BLASTOCYST DEVELOPMENT AFTER TRANSPLANTATION IN THE RABBIT
}

The viability of rabbit blastocysts studied at $5 \mathrm{I} / 2$ days post coitum was estimated from their size at that stage, rapidity of in vitro growth and oxygen consumption.

Transplantation of the blastocysts into $5 \mathrm{I} / 2$-day pregnant rabbits yielded an embryonic survival rate of $26 \mathrm{p}$. Ioo at day 28 of gestation. The high mortality was due to manipulation of the transplanted blastocysts and to the opening of the uterine horn.

Survival is significantly better for large-size blastocysts and for those which have the highest oxygen consumption (oxygen consumption expressed by the ratio $\frac{\text { quantity of oxygen consumed }}{\text { size }}$ )

\section{RÉFÉRENCES BIBLIOGRAPHIQUES}

Adams C. E., 1960. Studies on prenatal mortality in the rabbit Oryctolagus cuniculus : The amount and distribution of loss before and after implantation. J. Endoc., 19, 325-344.

Adams C. E., I97o. The development of rabbit eggs after culture in vitro for I-4 days. J. Embryol. exp. Morph., 23, 2I-34.

AdAms C. E., Eckstein P., 1965. The effect of intrauterine foreign bodies on pregnancy in the rabbit. Fert. Steril., 16, 508-521.

Dickson A. D., I966. The form of the mouse blastocyst. J. Anat., 100, 335-348.

Gates A. H., I965. Rate of ovular development as a factor in embryonic survival. In : Preimplantation stages of Pregnancy, Ciba Found. Symp.

Hafez E. S. E., 1962. "Differential cleavage rate" in 2-day litter mate rabbit embryos. Proc. Soc. Exp. Biol. Med., 110, 142-145.

STAPLES R. E., I97I. Blastocyst transplantation in the rabbit in : Methods in Mammalian Embryology, éd. Daniel J,-C. Jr, Freeman and Co San Francisco.

Wintenberger-Torrks S., I97o. Etude de la respiration des blastocystes de Lapine en culture in vitro. Ann. Biol. anim. Bioch. Biophys., 10, 187-194.

Wintenberger-Torrès S., Danel M. C., I97I. Etude du développement in vitro des blastocystes de Lapin. Ann. Biol. anim. Biach. Biophys., 11, 379-387. 